\title{
Simulation of Active Reflection Coefficient Phenomena of Large Antenna Array using FEM Domain Decomposition Methods
}

\author{
A. Barka, A. Jouadé, D. Jacquinot
}

\begin{abstract}
This paper proposes the application of the Finite Element Tearing and Interconnecting method (FETI-2LM) a Domain Decompositon Method (DDM) [1], [2] for the full-wave simulation of large antenna array (few thousands of radiating elements). The proposed methodology makes it possible to efficiently calculate in a post-processing step the antenna patterns for steering directions within the desired field of view while taking into account the active reflection coefficients. The methodology is based on an efficient extraction of the embedded radiating elements far field radiation patterns as well as the Generalized Scattering Matrix (GSM) [3] of the array by implementing massively parallel computers. The beauty of the GSM is its capability to handle the active S-parameters independently of the steering direction. This permits to generate the angulardependent active reflection coefficients by post-processing. This implementation is a step forward to provide us with a tool that permits to evaluate by simulation large antenna array while taking into account all the phenomena and interactions that may degrade the key performances that are required at a higher level, to specify a global system before fabrication.
\end{abstract}

Index Terms-Finite element, Domain Decomposition Method, Parallel machines, Finite Element Tearing and Interconnecting (FETI), Active Reflection Coefficient, Antenna Array

\section{INTRODUCTION}

$\mathbf{T}$ HE mutual couplings between the radiating elements need to be taken into account in order to achieve a proper modeling of an antenna array. These couplings can be responsible of blind directions for which the reflection coefficient is close to 1 causing a drop in the gain of the antenna in these singular directions. Taking into account the effects of active reflection coefficients during simulations of electromagnetic performances of an antenna array is therefore essential. This indicator allows to optimize either the antenna element itself but also the geometry of the array to reduce blind zone effects. These simulations performed with full-wave methods based on integral equations (EFIE, CFIE, MLFMM) [4] or partial differential equations (FEM finite element method) [5] can be extremely tedious and sometimes require the availability of very large computational resources. Several published works have demonstrated the effectiveness of exact or approximate numerical methods during parametric studies, based on domain decomposition approaches to characterize the antenna array as

This work was supported in part by the Grand Equipement National de Calcul Intensif (GENCI) Grant c2014109083.

A. Barka and A. Jouadé are with ONERA/DEMR, Université de Toulouse, France. ONERA is the French Aerospace Lab and DEMR its ElectroMagnetism and Radar Department. (e-mail: andre.barka@onera.fr, antoine.jouade@onera.fr). D. Jacquinot is with ADC Antenna Design Concept. well as all its electronic components (amplifiers, attenuators, power splitters and phase shifters) and $S$ parameters effects on the active reflection coefficient. For example ONERA [6], [7] and XLIM University of Limoges [8]-[11] have shown the advantage of characterizing an antenna array by on the one hand the set of embedded radiation diagrams and on the other hand the Generalized Scattering Matrix (GSM) of the array. This then makes it possible to calculate by linear recombinations the antenna diagrams for scanning in azimuth and elevation without having to repeat the $3 \mathrm{D}$ simulations. These techniques make it possible to take into account finely the effect of the active reflection coefficients during the beam steering.

The method proposed in [10], [11], by taking advantage of existing formalisms on periodic arrays [3], [12], [13] and by using appropriate methods to efficiently exploit such formulations, accurately defines the performances of all building blocks, from the feeding circuits to the radiating elements, over a frequency range. However, the methodology to extract the scattering matrix of the array is based on a mix of periodic approximations (for the center elements) and partial local embedded zones for the border elements of the array.

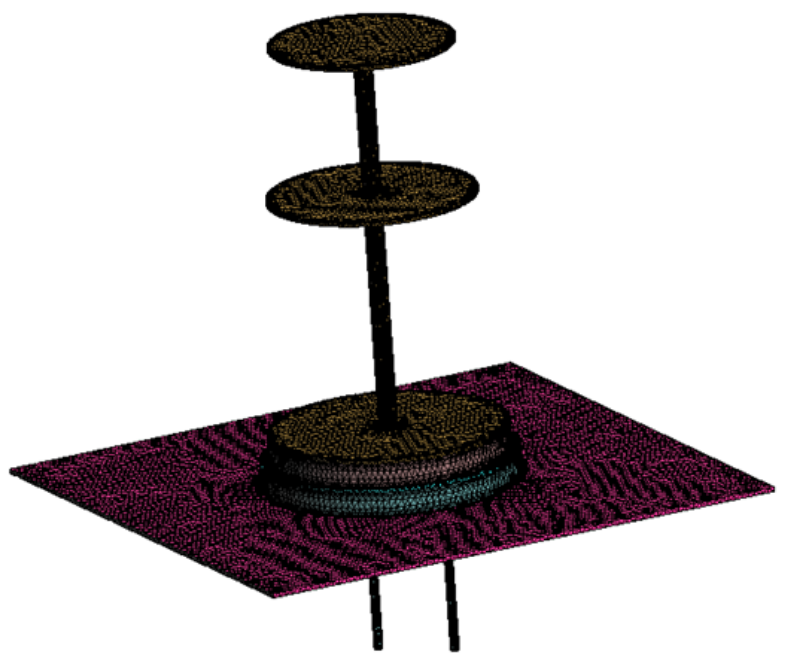

Fig. 1: Back-fire antenna geometry.

In this work, so as to efficiently calculate the embedded radiation patterns of all the radiating elements (internal and 
boundary radiating elements) as well as the complete Scattering Matrix [6] of the array, we discuss the interest of the implementation of the FETI-2LM domain decomposition methods taking into account the finitude o $\mathrm{ft}$ he a rray on massively parallel computers initially developed in [14] and optimized in [15] and [16] for large size sparse arrays. The main goal is to implement a method allowing in a first step to calculate the radiating elements radiation patterns and the GSM of the array, and in a second post processing step to calculate the gain patterns of the antenna array for azimuth and elevation scanning directions while taking into account the active reflection c oefficient. Th ese di agrams ca $n$ be quickly obtained with a very reduced computational effort, while offering an exact solution of the complete problem. The DDM method will be fully described in Section II, while Section III contains the obtained results from a 1600-elements antenna array.

\section{DOMAIN DECOMPOSITION FOR GSM EXTRACTION}

\section{A. Step by step description}

1) Field expansion on the $n^{\text {th }}$ TEM ports of the array: On each antenna port, the electric and magnetic fields $\left(E_{n}\right.$ and $\left.H_{n}\right)$ are expanding using incoming $\left(e_{n}, h_{n}\right)$ and outgoing $\left(e_{n},-h_{n}\right)$ TEM electromagnetic modal waves [17] of amplitudes $a_{n}$ and $b_{n}$.

$$
\begin{aligned}
& \mathbf{E}_{\mathbf{n}}=\left(\mathbf{a}_{\mathbf{n}}+\mathbf{b}_{\mathbf{n}}\right) \cdot \mathbf{e}_{\mathbf{n}} \\
& \mathbf{H}_{\mathbf{n}}=\left(\mathbf{a}_{\mathbf{n}}-\mathbf{b}_{\mathbf{n}}\right) \cdot \mathbf{h}_{\mathbf{n}}
\end{aligned}
$$

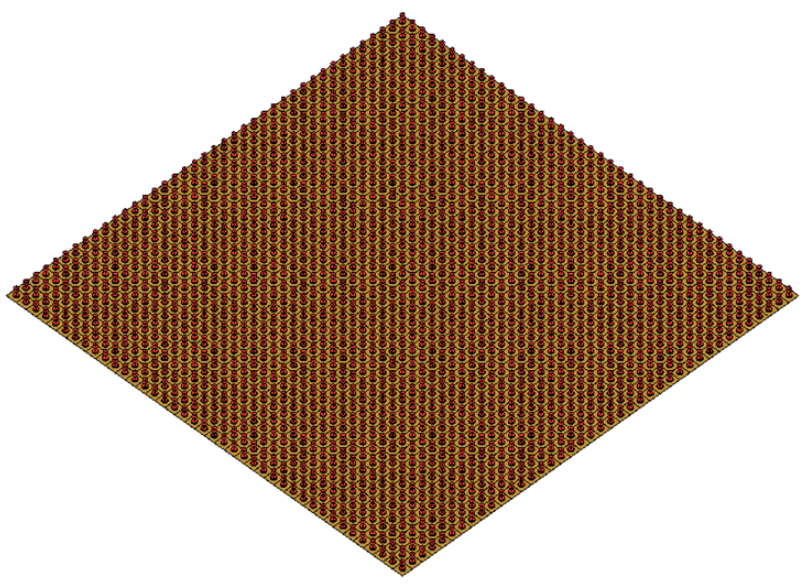

Fig. 2: Regular array of 1600 elements.

2) Solving the FEM problem: The Generalized Admittance Matrix (GAM) of the array is first FEM-computed with the FETI-2LM domain decomposition method [14] and converted to a Generalized Scattering Matrix (GSM) ' $S$ ' as proposed in [6].

$$
\mathbf{b}=\mathbf{S} \cdot \mathbf{a}
$$

This step is time and memory consuming and is performed on parallel clusters. The antenna array ports are fed and the simulation outputs are the GAM of the array and the complex radiation patterns $\tilde{\mathbf{e}}(\theta, \phi)$ of each antenna surrounded by the others.

3) Beamsteering: The beam-steering of the main lobe in a direction $\left(\theta_{p}, \phi_{p}\right)$ is performed by defining an amplitude and phase distribution $a_{n}\left(\theta_{p}, \phi_{p}\right)$ on each port of the array. The amplitudes of the outgoing waves $b_{n}$ re-entring in the antenna array due to mutual coupling are expressed through the GSM and equation 3. Finally, the electric farfield radiation pattern of an array of $\mathrm{N}$ elements for a $\left(\theta_{p}, \phi_{p}\right)$ beam direction is expressed by:

$$
\tilde{\mathbf{E}}_{(\theta, \phi)}=\sum_{n=1}^{N}\left(\mathbf{a}_{\mathbf{n}}\left(\theta_{\mathbf{p}}, \phi_{\mathbf{p}}\right)-\mathbf{S} \times \mathbf{a}_{\mathbf{n}}\left(\theta_{\mathbf{p}}, \phi_{\mathbf{p}}\right)\right) \tilde{\mathbf{e}}_{\mathbf{n}}(\theta, \phi)
$$

\section{B. Active reflection coefficients}

Hence, this calculation gives us the $\mathrm{n} \times \mathrm{n}$ scattering matrix of the radiating array. The active reflection coefficients of the elements located in this finite array can then be computed

$$
\mathrm{S}_{\mathrm{i}, \mathrm{i}}^{\text {active }}=\frac{1}{\mathrm{a}_{\mathbf{i}}} \sum_{\mathrm{j}=1}^{\mathrm{n}} \mathrm{S}_{\mathrm{ij}} \cdot \mathbf{a}_{\mathrm{j}}
$$

and the global active reflection coefficient of the array is:

$$
\mathrm{S}_{\text {array }}^{\text {active }}=\frac{1}{\mathrm{~N}} \sum_{\mathbf{i}=\mathbf{1}}^{\mathrm{N}} \mathrm{S}_{\mathbf{i}, \mathbf{i}}^{\text {active }}
$$

\section{GSM and Gain parallel simulation on HPC clusters}

We used the strategy proposed in a recent work [15] for sparse arrays, here by populating our regular grid of regular periodicity with two types of sub-domains (antenna and PEC extension of the arrays as shown on Fig. 2). Since the method is implemented on parallel machines, each computing core with at least $4 \mathrm{~GB}$ of memory is allocated exclusively to a sub-domain (elementary cell of the dense array). During the simulation we used 1764 sub-domains (1600 antenna subdomains and 164 border PEC extension of the array). Each of the 1600 radiating elements is excited from its TEM port and the nearfield solution is exported on all the 1600 TEM ports of the array. The simulation is speed up by using the block Krylov recycling algorithms developped in [14]. Finally the GAM and the GSM are calculated in a post processing step following the methodology of [6]. By denoted by $Z_{0}$ the free space wave impedance and by $P_{r}$ the radiated power of the antenna, the absolute gain of the array including the active reflection coefficients is obtained from the electric farfield defined by equation 4 and:

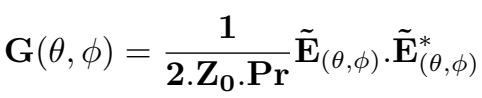

\section{RESULTS}

As an example, an array of 1600 elements covering 420 $m^{2}$ in a regular grid is shown in Fig. 2. The computational statistics are indicated in Table I. The full wave simulation is performed with the FACTOPO code using 1764 Intel Xeon 
Broadwell E5-2680v4 computing cores. During the simulation, each of the 1600 radiating elements is assigned to a core processor following the strategy proposed in [15]. Note that the simulation time for one embedded antenna is 9 minutes and the total simulation time to extract the complete GSM of the array (Figure 3) and the 1600 embedded diagrams is 240 hours. The antenna element is a back-fire antenna (Fig. 1) with 3 circular patches along the $\mathrm{z}$-direction working at around $440 \mathrm{MHz}$ and backed by a conducting ground plane. Such an antenna is a nice candidate for antenna array because it permits to have a high gain while having a low footprint along the array thanks to the use of the $3^{r d}$ dimension (z-direction). This antenna permits to reduce coupling effects with the neighbours to avoid strong blind zones thanks to lower active reflection coefficients.

TABLE I: Computational statistics

\begin{tabular}{|l|r|}
\hline Mesh parameters (unknowns) & Previous strategy \\
\hline Antenna sub-domain & 586,461 \\
\hline Skeleton interface problem & 13 millions \\
\hline Total unknowns of the array & 1 billion \\
\hline Simulation parameters & 1,764 \\
\hline Number of cores & 99 \\
\hline Number of iterations & $\epsilon=10^{-2}$ \\
\hline Stopping criterion & 9 minutes \\
\hline Elapse time (per antenna) & 240 hours \\
\hline Elapse time (complete 1600x1600 GSM) & $2.3 \mathrm{~Gb}$ \\
\hline Memory peak (per core) & \\
\hline
\end{tabular}

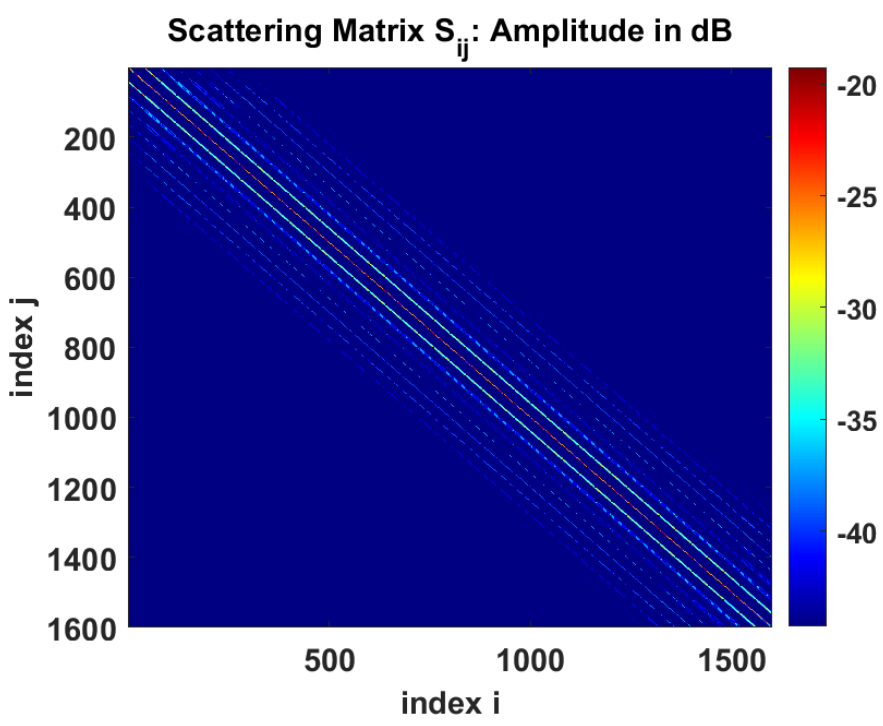

Fig. 3: Simulated GSM of the 1600x1600 antenna array

Fig. 4 shows the simulated reflection coefficient of the isolated antenna element using the FACTOPO code and in Figure 5, the simulated radiation patterns are shown along $\phi=0^{\circ}$ and $\phi=90^{\circ}$ respectively. Such an antenna has a frequency bandwidth of about $35 \mathrm{MHz}$ around a center frequency of $440 \mathrm{MHz}$. At the center frequency, the gain of this antenna is $10.5 \mathrm{dBi}$ with side lobe level of $-20 \mathrm{~dB}$ from the main lobe. The half-power beamwidths are $45^{\circ}$ along $\phi=0^{\circ}$ and $40^{\circ}$ along $\phi=90^{\circ}$.

Fig. 6 shows the active reflection coefficient of each element of the radiating panel. These coefficients are computed for a

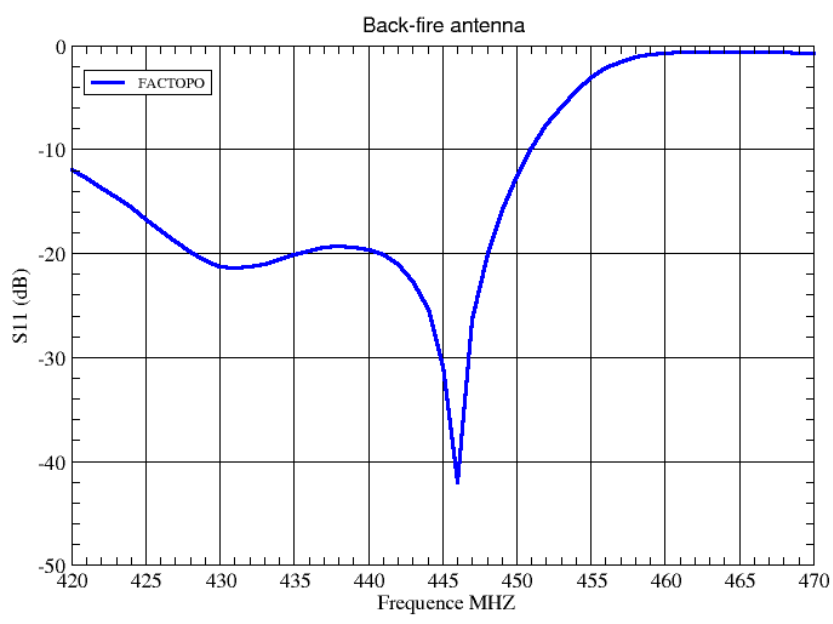

Fig. 4: Simulated reflection coefficient of the isolated element with its surrounded metallic ground.

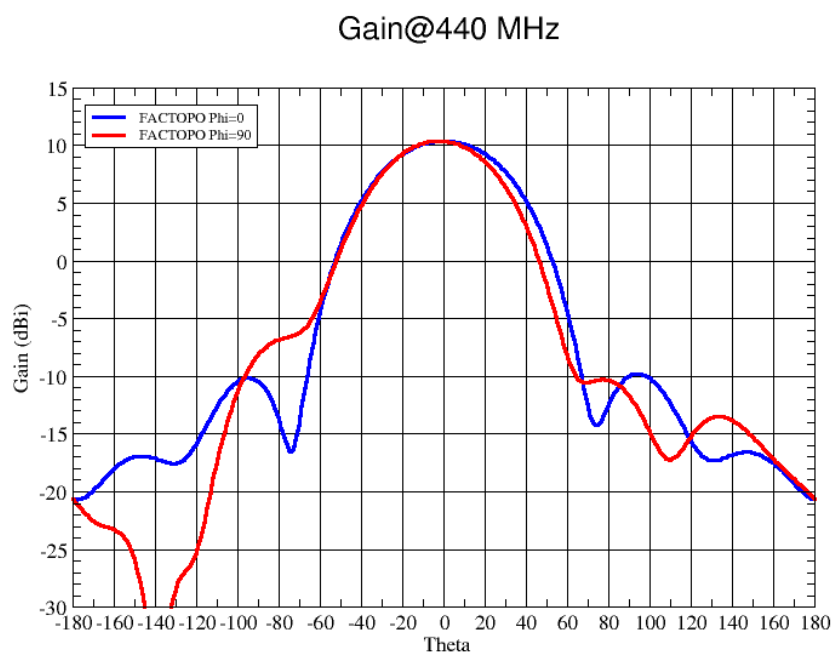

Fig. 5: Simulated radiation pattern of the isolated back-fire antenna along $\phi=0^{\circ}$ and $\phi=90^{\circ}$.

uniform amplitude excitation and a linear phase distribution corresponding to a specific beam steering (Azimuth $=15^{\circ}$ and Elevation $=0^{\circ}$ ), while in Fig. 7 is shown the radiation pattern of the array for the considered beam steering taking into account the active reflection coefficient. Finally, it is shown the variability of the active reflection coefficients of the global panel depending on the beamsteering considered. It can be shown an increase of the active reflection coefficients for steering angles higher than $25^{\circ}$ in Azimuth and $25^{\circ}$ in Elevation.

\section{CONCLUSION}

In this paper, the capability of calculating the active reflection coefficients of large arrays over a global field of view, and 


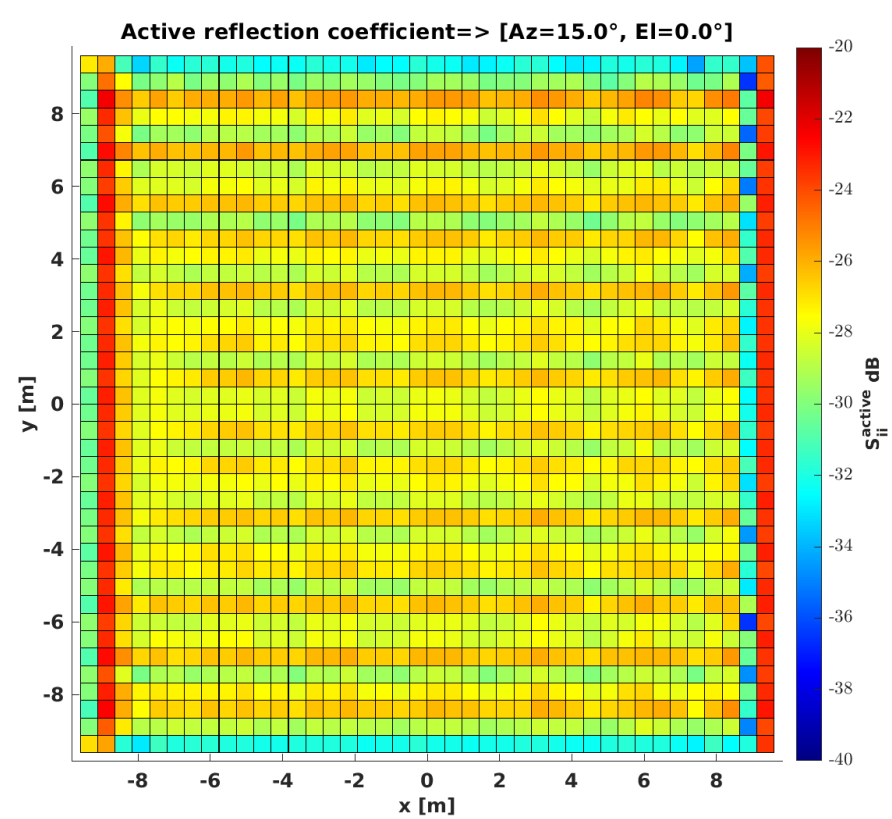

Fig. 6: Active reflection coefficients of each element of the array at one steering angle.

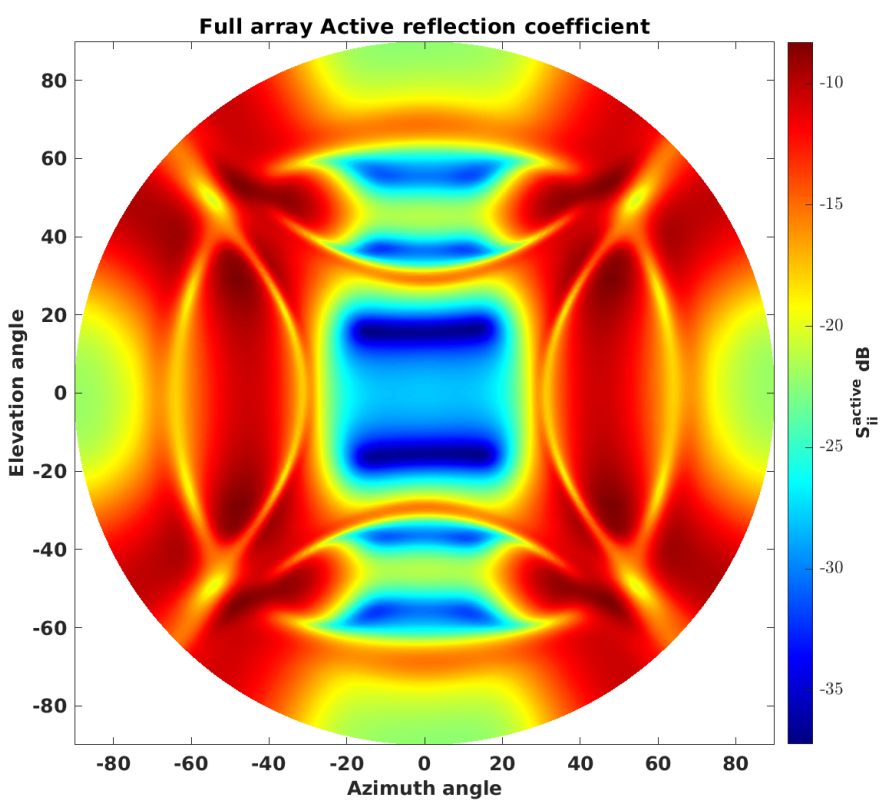

Fig. 7: Global active reflection coefficient of the array for all steering angles.

in a postprocessing step, has been demonstrated. Associated with the FETI-2LM Domain Decomposition Methods, this strategy permits to take into account all the phenomena and interactions that may degrade the key performances of antenna array. This development is a valuable step forward for future developments in antenna and array designs.

\section{REFERENCES}

[1] Y. Li and J.-M. Jin, "A vector dual-primal finite element tearing and interconnecting method for solving 3-d large scale elemectromagnetic problems," IEEE Trans. Antennas and Propagation, vol. 54, pp. 721733, Oct 2006.

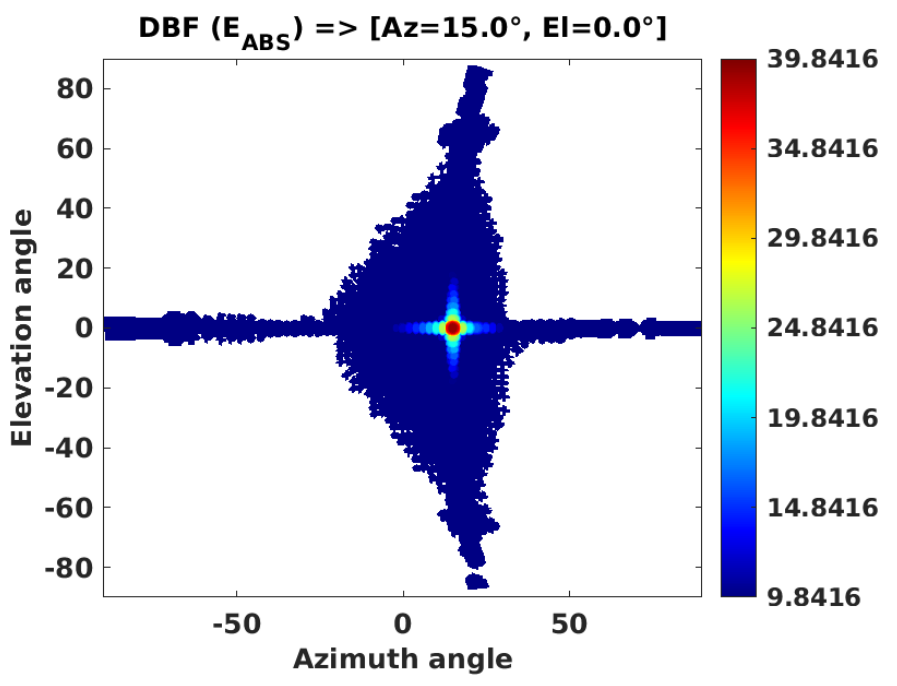

Fig. 8: Radiation pattern of the array in a specific direction.

[2] K. Zhao, V. Rawat, S. Lee, and J. Lee, "A domain decomposition method with nonconformal meshes for finite periodic and semi-periodic structures," IEEE Trans. Antennas and Propagation, vol. 55, pp. 25592570, Sept 2007.

[3] R. Mailloux, "Phased array antenna handbook, norwood, ma: Artech house," 2005 .

[4] J. Song, C. Lu, and W. Chew, "Multilevel fast-multiple algorithm for solving combined field integral equations of electromagnetic scattering," Compte rendu de l'Acad. Sci, CR Phys., vol. 7, pp. 474-485, Sept 1995.

[5] J. Jin, The Finite Element Method in Electromagnetics. New York Wiley, second ed., 2002.

[6] A. Barka and P. Caudrillier, "Domain decomposition method based on generalized scattering matrix for installed performance of antennas on aircraft," IEEE Transactions on Antennas and Propagation, vol. 55, no. 6, pp. 1833-1842, June 2017.

[7] M. Romier, A. Barka, H. Aubert, J.-P. Martinaud, and M. Soiron, "Load-pull effect on radiation characteristics of active antennas," IEEE Antennas and Wireless Propagation Letters, vol. 7, pp. 550-552, 2008.

[8] B. A, "Accuracy of floquet model in predicting performances of finite arrays," IEEE Antennas Wireless Propag. Lett., pp. 19-22, 2014.

[9] M. A, C. Menudier, Thevenot, F. Torres, and T. Monediere, "Determination of the scattering matrix of large periodic antenna arrays," in Proc. 10th Eur. Conf. Antennas Propag. (EuCAP), Davos, Switzerland,, 2016.

[10] M. Thevenot and al, "Synthesis of antenna arrays and parasitic antenna arrays with mutual couplings," Int. J. Antennas Propag., vol. 2012, 2012.

[11] B. Lesur and all, "A large antenna array for ka-band satcom-onthe- move applicationsaccurate modeling and experimental characterization," IEEE Transactions on Antennas and Propagation, vol. 66, no. 9, pp. 45864594, 2018.

[12] A. K. Bhattacharyya, "Accuracy of floquet model in predicting performances of finite arrays," IEEE Antennas and Wireless Propagation Letters, vol. 13, pp. 19-22, 2013.

[13] A. Maati, C. Menudier, M. Thevenot, F. Torres, and T. Monediere, "Determination of the scattering matrix of large periodic antenna arrays," in 2016 10th European Conference on Antennas and Propagation (EuCAP), pp. 1-3, IEEE, 2016.

[14] F.-X. Roux and A. Barka, "Block krylov recycling algorithms for feti$21 \mathrm{~m}$ applied to 3-d electromagnetic wave scattering and radiation," IEEE Transactions on Antennas and Propagation, vol. 65, no. 4, pp. 1886$1895,2017$.

[15] A. Jouadé and A. Barka, "Massively parallel implementation of feti-2lm methods for the simulation of the sparse receiving array evolution of the graves radar system for space surveillance and tracking," IEEE Access, vol. 7, pp. 128968-128979, 2019.

[16] A. Barka and A. Jouadé, "Improvement of ddm pre-processing for the simulation of the graves radar densified sparse array for space surveillance and tracking," IEEE Antennas and Wireless Propagation Letters, p. DOI 10.1109/LAWP.2020.3004925, July 2020.

[17] P. Silvester and R. Ferrari, Finite Elements for Electrical Engineers. Cambridge, MA: Cambridge Univ. Press, second ed., 1990. 